\title{
Effectiveness of prospective audit-and-feedback intervention in promoting appropriate third- generation cephalosporin use at a tertiary public hospital in Malaysia
}

Rou Wei Tan ( $\square$ rouwei90@hotmail.com )

Hospital Raja Permaisuri Bainun

Kah Shuen Thong

Hospital Raja Permaisuri Bainun

Chee Tao Chang ( $\square$ davidcct.crc@gmail.com )

Hospital Raja Permaisuri Bainun https://orcid.org/0000-0002-9263-446X

Joo Thye Cheng

Hospital Raja Permaisuri Bainun

Huan Keat Chan

Hospital Raja Permaisuri Bainun

Meng Fei Cheah

Hospital Raja Permaisuri Bainun

\section{Research Article}

Keywords: Cephalosporins, antimicrobial stewardship, feedback, inappropriate prescribing, nonadherence, Malaysia

Posted Date: March 24th, 2021

DOI: https://doi.org/10.21203/rs.3.rs-337913/v1

License: (c) (1) This work is licensed under a Creative Commons Attribution 4.0 International License. Read Full License 


\section{Abstract}

Background: Inappropriate use of broad-spectrum antibiotics accelerates the development of drug resistance in nosocomial pathogens. In Malaysia, the National Antimicrobial Guidelines (NAG), along with the feedback from antimicrobial stewardship (AMS) teams, serves as an important guide for antibiotic use in hospitals.

Objectives: This study aimed to determine the effectiveness of a prospective audit-and-feedback intervention in promoting the appropriate prescribing of third-generation cephalosporins and timely culture and sensitivity (C\&S) testing in patients admitted to a neurosurgical ward.

Method: In the pre-intervention phase, a ward pharmacist examined medical records of the patients receiving treatment with third-generation cephalosporins. The use of a cephalosporin was deemed to be appropriate only if it was in line with either the NAG or the recommendations made by the AMS team. The laboratory reports were also checked to see if the C\&S testing was performed right before the first dose of a cephalosporin was given. The findings were subsequently presented and discussed in a 2-hour feedback session. The post-intervention audit was performed in the same manner as in the preintervention phase.

Results: Seventy cases were studied in the pre- and post-intervention phases each. The proportion of cases with appropriate use of third-generation cephalosporin increased significantly from $77.1 \%(54 / 70)$ to $95.8 \%(67 / 70)$ following the intervention $(p=0.001)$. The proportion of cases with a C\&S test performed timely also increased significantly from $38.6 \%(27 / 70)$ to $58.6 \%(41 / 70)(p=0.018)$.

Conclusion: The prospective audit-and-feedback intervention was effective in improving the appropriateness of the prescribing of third-generation cephalosporins and timely culture and sensitivity testing, indicating the antimicrobial stewardship strategy had produced a positive outcome.

\section{Introduction}

Third-generation cephalosporins generally have a broad spectrum of antimicrobial activities and are able to cross the blood-brain barrier. This gives rise to their high usage as the empirical treatment of neurosurgical complications [1, 2], which is often linked to antimicrobial resistance in nosocomial pathogens [3]. As a result, the treatment options for infections caused by gram-negative bacteria has become limited [4].

In Asia, ceftriaxone is the most widely used third-generation cephalosporin, prescribed for $59.1-81.8 \%$ of hospitalized patients [5,6]. Most of the time, it was used as the empirical treatment [7], including in neurosurgical wards [8]. Cefoperazone/ sulbactam came in second, mainly prescribed for those with sepsis [9]. 
In Malaysia, the cephalosporin usage reached 650 defined daily dose/ 1000 patient-days in 2016 . Slightly less than $80 \%$ of the cases had cephalosporins used as the empirical treatment, $13.6 \%$ for microbiologically confirmed diseases and $5.7 \%$ as surgical prophylaxis [10]. However, the use of antibiotics in wards was commonly found to be inconsistent with the recommendations in the National Antimicrobial Guidelines (NAG) [11]. Another study also related the inappropriate antibiotic prescribing to growing antimicrobial resistance and mortality in hospitalized patients [12]. It is believed that the most effective way to combat antimicrobial resistance is through the rational use of antibiotics [13].

The Antimicrobial Stewardship (AMS) Program was launched by the Ministry of Health $(\mathrm{MOH})$ to promote the appropriate prescribing of antibiotics in Malaysia. It is run by a multidisciplinary team in each hospital, which includes infectious disease physicians, clinical microbiologists and ward pharmacists [14]. One of the important activities of the AMS team is to perform audits and provide feedback [15], with the aim to correct the inappropriate practice in antibiotic use [16].

Together with the AMS program, the NAG was introduced to guide the prescribers in antibiotic use. In public health institutions in Malaysia, prescribers are expected to follow the recommendations of the NAG when it comes to antibiotic use. The adherence to the NAG could be evaluated in several aspects, including the drug selection, the regimen used, and the purpose of treatment. Meanwhile, the AMS team served as an important point of reference when a clinical condition was not captured by the NAG.

In the Raja Permaisuri Bainun Hospital located in northern Malaysia, the 38-bed neurosurgical ward recorded the highest usage of ceftriaxone, with a defined daily dose of 189.11/1000 bed days between January and June 2019. This study aimed to evaluate the effectiveness of an audit-and-feedback intervention in promoting the appropriate prescribing of third-generation cephalosporins and timely culture and sensitivity (C\&S) testing among patients admitted to this ward.

\section{Methods}

This audit consisted of the pre-intervention, feedback and post-intervention phases. The patients included were those who were admitted to the neurosurgical ward during the pre- and post-intervention of the audit and received treatment with one of the three commonly used third-generation cephalosporin (ceftriaxone, cefoperazone/sulbactam and ceftazidime).

The sample size required in each phase was estimated based on a previous study, which reported that the proportions of prescriptions showing an appropriate use of empirical antibiotics were, respectively, $61.7 \%$ and $83.8 \%$ before and after an intervention (15). With the confidence level set at $95 \%$ and power at $80 \%$, the calculated sample size was 63 in the pre- and post-intervention phases each. To account for $10 \%$ of cases with incomplete information, 70 cases were studied in both phases.

\section{Pre-intervention phase ( $1^{\text {st }}$ July $-31^{\text {st }}$ October 2019)}


A ward pharmacist, who was also the AMS pharmacist, extracted the data from medical records of patients treated with a third-generation cephalosporin between $1^{\text {st }}$ July and $31^{\text {st }}$ October 2019 . The data collection and assessment were conducted at the point when the first dose of a cephalosporin was administered prior to any interventions made by the ward pharmacist. The information gathered included the types of antibiotics, their dosage and frequency, duration and the diagnosis of patients. In this context, inappropriate prescribing referred to the use of a cephalosporin not in line with either the NAG or recommendations of the AMS team, in term of drug selection, dose and frequency; and treatment duration. In a case that a condition was not captured by the NAG, the prescriber was expected to seek advices from the AMS team. Also, the prescribers were expected to perform a C\&S test right before the first dose of a cephalosporin was given.

The ethics approval for this study was obtained from the Medical Research and Ethics Committee (MREC) under the MOH, Malaysia [(Registration number: NMRR-19-804-47375 (IIR), approval number: KKM/NIHSEC/P19-945(10).

\section{Intervention phase $\left(13^{\text {th }}\right.$ December 2019)}

The audit findings were then presented in a 2-hour feedback session, in which all the cases of inappropriate prescribing were discussed. The feedback session was led by the hospital AMS team which consisted of AMS pharmacists, clinical microbiologists and infectious disease physicians. The attendees included neurosurgeons and medical officers from the neurosurgical department. The audit feedback was presented by the ward AMS pharmacist and the discussion emphasized the need to use cephalosporins according to the recommendations of the NAG (Table 1), as well as to perform C\&S testing right before empirical treatment was initiated.

Table 1

Summary note of recommended treatment for several common conditions in a neurosurgical ward (adapted from the National Antibiotics Guidelines 2019).

\begin{tabular}{|lll|}
\hline No. & Conditions & Recommended treatment \\
\hline $\mathbf{1}$ & Intracranial trauma and open fracture & $\begin{array}{l}\text { IV Cefuroxime 1.5g TDS + IV Metronidazole 500mg } \\
\text { TDS/PO Metronidazole 400mg TDS }\end{array}$ \\
$\mathbf{2}$ & $\begin{array}{l}\text { Depressed skull fracture and } \\
\text { penetrating craniocerebral injury }\end{array}$ & $\begin{array}{l}\text { IV Ceftriaxone 2g BD + IV Metronidazole 500mg } \\
\text { TDS/PO Metronidazole 400mg TDS }\end{array}$ \\
$\mathbf{3}$ & Aspiration pneumonia & IV Amoxycillin/Clavulanic Acid 1.2g TDS \\
$\mathbf{4}$ & $\begin{array}{l}\text { Hospital-acquired pneumonia } \\
\text { i. Early onset } \\
\text { ii. Late onset }\end{array}$ & $\begin{array}{l}\text { i. IV Augmentin 1.2 g TDS } \\
\text { ii. IV Piperacillin/Tazobactam 4.5g QID or IV Cefepime } \\
2 \mathrm{~g} \text { TDS }\end{array}$ \\
\hline
\end{tabular}

Note: IV = intravenous, TDS = three times daily, $\mathrm{PO}=$ per os (orally), $\mathrm{BD}=$ two times daily, $\mathrm{QID}=$ four times daily 
The neurosurgical team agreed to the above recommendations, with an exception given to the use of ceftriaxone at their discretion in patients who had a basilar skull fracture complicated with cerebrospinal fluid (CSF) leak.

\section{Post-intervention phase (15 ${ }^{\text {th }}$ December $2019-31^{\text {st }}$ August 2020)}

The post-intervention audit was performed in the same manner as in the pre-intervention phase. No staff change took place throughout the audit period. To minimize the Hawthorne effect [17], only the head of neurosurgical department was informed of the conduct of the audit. Only one ward pharmacist was involved in the data collection and assessment in order to ensure the internal reliability of the assessment.

The statistical analysis was performed using the SPSS for Windows (Version 20.0. Armonk, NY: IBM Corp). The categorical variables were described as frequencies and percentages, and continuous variables as mean with standard deviation (SD). The independent t- and the Pearson's chi-square tests were used to assess the differences in the patients before and after the intervention. The statistical significance of the test was indicated by a $p$-value $<0.05$.

\section{Results}

The medical records of 70 patients were examined in both the pre- and post-intervention phases. No differences in the distributions of age $(p=0.759)$ and gender $(p=0.847)$ were observed between two phases. Presumed meningitis was the most common conditions, reported for $44(62.9 \%)$ and 40 (57.2\%) patients in the pre- and post-intervention phases, respectively $(P=0.490)$. Hospital- acquired pneumonia was more found to be more common in the pre-intervention phase $(P=0.029)$, while more patients had brain abscess in the post-intervention phase $(P=0.009)$. Ceftriaxone was the most widely prescribed third-generation cephalosporin. It was given to 63 (89.9\%) and 68 (97.1\%) patients before and after intervention, respectively (Table 2$)$. There were no significant differences in length of stay $(p=0.063)$, thirty-day readmission $(p=0.822)$ and thirty-day mortality $(p=0.698)$ between the two groups of patients. 
Table 2

Characteristics of patients.

\begin{tabular}{|c|c|c|c|}
\hline Characteristics $(n, \%)$ & Pre-intervention & Post-intervention & P-value \\
\hline Age & $36.4 \pm 16.52$ & $37.3 \pm 18.63$ & 0.759 \\
\hline Gender & $53(75.7)$ & $51(72.9)$ & 0.847 \\
\hline Male & $17(24.3)$ & $19(27.1)$ & \\
\hline \multicolumn{4}{|l|}{ Female } \\
\hline Clinical Diagnosis & $44(62.9)$ & $40(57.2)$ & 0.490 \\
\hline \multicolumn{4}{|l|}{ Presumed meningitis (total) } \\
\hline SSI with osteomyelitic changes & $13(18.6)$ & $9(12.9)$ & 0.353 \\
\hline Pneumocephalus & $13(18.6)$ & $21(30.0)$ & 0.115 \\
\hline Depressed skull fracture & $11(15.7)$ & $2(2.9)$ & 0.009 \\
\hline Inner \& outer skull fracture & $3(4.3)$ & $3(4.3)$ & 1.000 \\
\hline Basilar skull fracture with CSF leak & $4(5.7)$ & $5(7.1)$ & 0.730 \\
\hline Brain abscess & $1(1.4)$ & 9 (12.9) & 0.009 \\
\hline Closed skull fracture & $1(1.4)$ & $1(1.4)$ & 1.000 \\
\hline Open skull fracture & $5(7.2)$ & - & - \\
\hline Surgical chemoprophylaxis & $3(4.3)$ & - & - \\
\hline Hospital acquired pneumonia & $6(8.6)$ & - & - \\
\hline Confirmed meningitis & $4(5.8)$ & $10(14.3)$ & 0.091 \\
\hline Ventriculitis & - & $1(1.4)$ & - \\
\hline Community acquired pneumonia & - & $1(1.4)$ & - \\
\hline Infected VP shunt & $1(1.4)$ & $3(4.3)$ & 0.310 \\
\hline Subdural empyema & $1(1.4)$ & $4(5.7)$ & 0.172 \\
\hline Occult sepsis & $1(1.4)$ & - & - \\
\hline Cerebral Toxoplasmosis & $1(1.4)$ & - & - \\
\hline Penetrating injury & $1(1.4)$ & - & - \\
\hline Scalp abscess & - & $1(1.4)$ & - \\
\hline Aspiration pneumonia & $1(1.4)$ & - & - \\
\hline Note: SSI = surgical site infection & & & \\
\hline
\end{tabular}




\begin{tabular}{|llll|}
\hline Characteristics $(\mathrm{n}, \%)$ & Pre-intervention & Post-intervention & P-value \\
\hline Antibiotics used & $63(90)$ & $68(97.2)$ & 0.007 \\
Ceftriaxone & $7(10)$ & - & \\
Cefoperazone/sulbactam & - & $2(2.8)$ & \\
Ceftazidime & & & \\
Length of stay, mean (SD) & $11.79(11.26)$ & $16.23(16.33)$ & 0.063 \\
\hline Thirty days readmission & $4(5.7)$ & $7(10.0)$ & 0.822 \\
\hline Thirty days mortality & $3(4.3)$ & $4(5.7)$ & 0.698 \\
\hline Note: SSI = surgical site infection & & & \\
\hline
\end{tabular}

The proportion of cases with an appropriate use of third-generation cephalosporins had significantly increased from $77.1 \%(54 / 70)$ to $95.8 \%(67 / 70)$ following the intervention $(p=0.001)$. Common issues detected in the pre-intervention phase included an inappropriate selection of antibiotics ( $n=13 / 70$, $18.7 \%)$ and an inappropriate treatment duration $(n=1 / 70,1.4 \%)$. Of the 13 cases with an inappropriate antibiotic selection, five were open skull fracture and six were hospital-acquired pneumonia. After the intervention, an inappropriate selection of antibiotics was only observed in two cases (Table 3).

Additionally, there were three cases ( 2 before the intervention and 1 after the intervention) in which the use of antibiotics was not recommended in the NAG and the prescriber did not consult the AMS team beforehand. 
Table 3

Findings of pre- and post-intervention audits.

\begin{tabular}{|c|c|c|c|}
\hline Characteristics & Pre-intervention & Post-intervention & P-value \\
\hline Prescribing Issues (Total) & $16(22.9)$ & $3(4.3)$ & 0.001 \\
\hline Inappropriate drug selection & $13(18.7)$ & $2(2.8)$ & 0.003 \\
\hline Inappropriate treatment duration & $1(1.4)$ & - & - \\
\hline Indication not stated in NAG* & $2(2.8)$ & $1(1.4)$ & 0.559 \\
\hline Conditions involved & $1(6.3)$ & - & NA \\
\hline Mastoid fracture & 5 (31.3) & - & \\
\hline Open skull fracture & $1(6.3)$ & - & \\
\hline Surgical prophylaxis & $1(6.3)$ & - & \\
\hline Cerebral toxoplasmosis & $1(6.3)$ & - & \\
\hline Occult sepsis & $6(37.2)$ & - & \\
\hline Hospital acquired pneumonia & $1(6.3)$ & - & \\
\hline Aspiration pneumonia & - & $1(33.3)$ & \\
\hline Close skull fracture & - & $1(33.3)$ & \\
\hline Community acquired pneumonia & - & $1(33.3)$ & \\
\hline \multicolumn{4}{|l|}{ Scalp abscess } \\
\hline Culture and sensitivity test & $43(61.4)$ & $29(41.4)$ & 0.018 \\
\hline Not done timely & $27(38.6)$ & $41(58.6)$ & \\
\hline Done timely & $7(10.0)$ & $22(31.5)$ & \\
\hline CSF & $7(10.0)$ & $1(1.4)$ & \\
\hline Blood & $8(11.4)$ & $14(20.0)$ & \\
\hline Tissue & $5(7.2)$ & $4(5.7)$ & \\
\hline \multicolumn{4}{|l|}{ Swab } \\
\hline Type of treatment involved & $67(95.7)$ & $69(98.6)$ & 0.245 \\
\hline Empirical & $3(4.3)$ & - & \\
\hline Surgical Prophylaxis & - & $1(1.4)$ & \\
\hline Definitive & & & \\
\hline
\end{tabular}


The proportion of cases with of a C\&S test performed timely also increased significantly from $38.6 \%$ $(27 / 70)$ to $58.6 \%(41 / 70)$ following the intervention $(p=0.018)($ Table 3$)$.

\section{Discussion}

This study shows that prospective audit-and-feedback intervention can serve as an effective approach to improve the appropriateness of third-generation cephalosporin prescribing in a hospital. It could also be used to promote the practice of performing the C\&S tests timely, which are essential to guide the rational use of antibiotics in the ward. Additionally, the 30-day mortality, 30-day readmission and length of stay in hospital was similar in both groups of patients, suggesting the safety of our intervention.

As it would be expected, ceftriaxone emerged as the most commonly prescribed third-generation cephalosporin in the ward. In both the pre- and post-intervention phases, most patients received it as the empirical treatment for presumed bacterial meningitis. A previous study supported such a practice, suggesting that prompt treatment for presumptive meningitis with broad-spectrum antibiotics could lower the risk of mortality [18]. The latest evidence also recommends the use of either ceftriaxone or cefotaxime as the empirical treatment for the same condition [19]. It is noteworthy that we did not seek to change the existing practice but advocated for the use of antibiotics with a narrow spectrum of antimicrobial activities for other clinical conditions, such as intracranial trauma, open fracture and depressed skull fracture, as recommended by the NAG (Table 1).

It was also found that six patients with hospital-acquired pneumonia and one patient with aspiration pneumonia were treated with cefoperazone/ sulbactam. However, the NAG recommends the use of amoxicillin/ clavulanate for early onset of both the conditions instead [19]. Such discrepancies were highlighted in the feedback session, and the appropriate drug selection for both the conditions were discussed. The prescribers adhered to this recommendation in the post-intervention phase, and no patient received cefoperazone/sulbactam for similar conditions. This suggests the prescribers have high level of acceptance towards evidence-based recommendations made by the AMS team.

There were also a few cases of basilar skull fracture complicated with CSF leak in both phases, for which antimicrobial treatment is not recommended by the NAG. However, the neurosurgical team raised their concern that the CSF leak could elevate the risk of meningitis. A consensus was reached in the feedback session that such a practice was acceptable. This shows a good example that the AMS team could play an integral role in providing timely and relevant advices when a condition is not captured by the NAG [15]. Similar conditions were also witnessed for cases of mastoid fracture, occult sepsis and close skull fracture.

In the pre-intervention phase, only 27 out of $70(38.6 \%)$ patients had the C\&S test performed timely. Ideally, the C\&S test is to be performed before the initiation of antimicrobial treatment [20]. However, such a practice is often hindered by the complexity lumbar puncture (LP) procedure, as patients need to undergo a CT scan first and those with septic shock are unfit for the procedure [21]. Right after the feedback session, we observed a significant increase in the number of C\&S tests performed timely. 
This was the first local study on the effectiveness of an audit-and-feedback intervention in promoting the appropriateness of third-generation cephalosporins use. Nevertheless, as this was a single center study, the findings are not generalizable to other clinical settings. This was also a one-off audit, and thus the sustainability of the change in prescribing patterns could not be evaluated.

\section{Conclusion}

The prospective audit-feedback intervention was shown to be effective in improving the appropriateness of the third-generation cephalosporin use and timely C\&S testing, indicating the antimicrobial stewardship strategy had produced a positive outcome. Further studies assessing the sustainability of the improvement are warranted.

\section{Declarations}

Ethics approval and consent to participate: This study was registered in the Malaysia National Medical Research Registry (NMRR-19-804-47375) and obtained the approval of Malaysian Medical Research and Ethics Committee (MREC). All research procedures were conducted according to the Malaysian Guidelines Good Clinical Practice ( $4^{\text {th }}$ edition) and other relevant guidelines for research. This was a prospective audit of case notes, hence informed consent was waived by the Ethical Committee.

Consent for publication: Not applicable

Availability of data and materials: The datasets generated and/or analysed during the current study are not publicly available due to confidentiality of patients, but are available from the corresponding author on reasonable request.

Competing Interest: The authors declare no conflict of interest.

Funding: This is a self-funded work.

Authors' contributions: TRW and TKS initiated the idea of this research work and drafted the proposal. TRW and CMF collected the data. CCT and CHK contributed to the data analysis and manuscript drafting. TKS and CJT provided administrative support and supervision. All authors proofread and approve the final version of this manuscript.

Acknowledgement: We would like to thank the Director General of Health Malaysia for his permission to publish this article, and Antimicrobial Stewardship Team Hospital Raja Permaisuri Bainun for all the guidance in conducting the study.

\section{References}

1. Buang SS, Haspani MS. Risk factors of neurosurgical site infections after a neurosurgical procedure: a prospective, observational study at Hospital Kuala Lumpur. Med J Malaysia. 2012;67(4):393-86. 
2. Guruja MP, Sarah A, Samaga L, Joshi H, Nair S, Shastry CS. Cephalosporin utilization evaluation in a university teaching hospital: a prospective study. Journal of Drug Delivery Therapeutics. 2013;3(2):83-7.

3. Mc Gowan JE, Tenover FC. Control of antimicrobial resistance in the health care system. infectious disease clinics of North America. 1997;11(2):297-311.

4. Park SH. Third generation cephalosporin resistance in gram-negative bacteria in the community: a growing public health concern. Korean Journal of International Medicine. 2014;29(1):27-30.

5. Abou-Shaaban, Ali AA, Rao PG, Majid A. Drug utilization review of cephalosporins in a secondary care hospital in United Arab Emirates. International Journal of Clinical Pharmacy. 2016;38(6):136771.

6. Naveen V, Siddiq A, Chandana G. A study of drug utilization pattern of cephalosporins in general medicine and surgical inpatient department. International Journal of Current Pharmaceutical Research. 2018;10(3):33-6.

7. Sileshi A, Tenna A, Feyissa M, Shibeshi W. (2016). Evaluation of ceftriaxone utilization in medical and emergency wards of Tikur Anbessa specialized hospital: A prospective cross-sectional study. BMC Pharmacology Toxicology, 17. https://doi.org/10.1186/s40360-016-0057-x.

8. Opanga SA, Nimrod JA, Faith AO, Kimani AM. Patterns of antimicrobial use in neurosurgical ward of Kenyatta National Hospital. African Journal of Pharmacology Therapeutics. 2016;5(4):241-6.

9. Sharma MS, Suri A, Chandra SP, Kale SS, Kapil A, Sharma BS, Mahapatra AK. Cost and usage pattern of antibiotics in a tertiary care of neurosurgical unit. Indian Journal of Neurosurgery. 2012;1(1):41-7.

10. Malaysian Action Plan on Antimicrobial Resistance. 2017-2021. Ministry of Health Malaysia and Ministry of Agriculture and Agro-based Industry Malaysia.

11. Lim MK, Lai PS, Ponnampalavanar SS, Syed Omar SF, Taib NA, Yusof MY, Italiano CM, Kong DC, Kamarulzaman A. Antibiotics in surgical wards: use or misuse? A newly industrialized country's perspective. J Infect Dev Ctries. 2015 Nov 30;9(11):1264-71. doi: 10.3855/jidc.6731. PMID: 26623636.

12. Gillani SW, Sulaiman AS, Nejad FB. Inpatient care and microbial surveillance during year 2007-2008; retrospective evaluation of hospital-acquired pneumonia (HAP) in General Hospital Pulau Pinang, Malaysia. International Journal of Food Safety Nutrition Public Health. 2010;3(1):27-32. https://doi.org/10.1504/IJFSNPH.2010.032032.

13. Parulekar L, Soman R, Singhal T, Rodrigues C, Dastur FD, et al. How good is compliance with surgical antibiotic prophylaxis guidelines in a tertiary care private hospital in India? A prospective study. Indian Journal of Surgery. 2009;71:15-8.

14. Ministry of Health Malaysia. Protocol on antimicrobial stewardship program in healthcare facilities. 2014. https://www.pharmacy.gov.my/v2/en/documents/protocolantimicrobial-stewardship-programhealthcare-facilities.html.

15. Høgli JU, Garcia BH, Skjold F, Skogen V, Småbrekke L. An audit-and-feedback intervention study increased adherence to antibiotic prescribing guidelines at a Norwegian hospital. BMC Infect Dis. 
2016 Feb 27;16:96. doi: 10.1186/s12879-016-1426-1. PMID: 26920549; PMCID: PMC4769530.

16. Ivers N, Jamtvedt G, Flottorp S, Young JM, Odgaard-Jensen J, French SD, O'Brien MA, Johansen M, Grimshaw J, Oxman AD. (2012). Audit-and-feedback: Effects on professional practice and healthcare outcomes. Cochrane Database of Systematic Reviews, 6.

https://doi.org/10.1002/14651858.CD000259.pub3.

17. McCambridge J, Witton J, Elbourne DR. Systematic review of the Hawthorne effect: new concepts are needed to study research participation effects. J Clin Epidemiol. 2014 Mar;67(3):267-77. doi:10.1016/j.jclinepi.2013.08.015. Epub 2013 Nov 22. PMID: 24275499; PMCID: PMC3969247.

18. Proulx N, Frechette D, Toye B, Chan J, Kravcik S. Delays in the administration of antibiotics are associated with mortality from adult acute bacterial meningitis. QJM. 2005;98:291-8.

19. Ministry of Health Malaysia. National Antimicrobial Guidelines (2019).

20. Young N, Thomas M. Meningitis in adults: Diagnosis and management. Internal Medicine Journal. 2018;48(11):1294-307. https://doi.org/10.1111/imj.14102.

21. McGill F, Heyderman RS, Michael BD, Defres S, Beeching NJ, Borrow R, et al. The UKjoint specialist societies guideline on the diagnosis and management of acute meningitis and meningococcal sepsis in immunocompetent adults. J Infect. 2016;72:405-38. 